\title{
Efficacy and Tolerability of a Medical Device Repairing Emollient Cream Associated with a Topical Corticosteroid in Adults with Atopic Dermatitis: An Open-label, Intra-individual Randomized Controlled Study
}

\author{
Ana Beatris Rossi • Adeline Bacquey • Thérèse Nocera • \\ Marie-Dominique Thouvenin
}

Received: January 8, 2018 / Published online: March 6, 2018

(C) The Author(s) 2018

\section{ABSTRACT}

Introduction: Medical device repairing emollient creams (MDRECs) are designed to repair and protect the skin barrier. In this study, we examined the added clinical benefit and tolerability of a MDREC when used in association with a moderately potent topical corticosteroid (TCS) for adults with atopic dermatitis (AD).

Methods: This was an intra-individual randomized controlled trial in adults with moderate to severe AD (EudraCT no. 2014-002,19410). Symmetrical lesions on each arm of the subjects were randomized to treatment for 10 days with twice-daily TCS (desonide) cream alone or with combined TCS + MDREC. Subjects were then included in a following 2-week

Enhanced content To view enhanced content for this article go to https://doi.org/10.6084/m9.figshare. 5853624

A. B. Rossi - A. Bacquey - T. Nocera .

M.-D. Thouvenin ( $\square)$

Clinical Skin Research and Development Center, Pierre Fabre Dermo-Cosmétique, 2 Rue Viguerie, Hotel Dieu, Toulouse, France

e-mail: marie.dominique.thouvenin@pierre-fabre.com

A. B. Rossi - T. Nocera

Department of Dermatology, University Hospital

Toulouse, 24, chemin de Pouvourville TSA, 31059

Toulouse CEDEX 9, France maintenance phase if the $\mathrm{AD}$ on at least one test area had sufficiently improved so that the treatment was no longer needed. During the maintenance phase, treatment with the TCS cream was stopped, but twice-daily application of the MDREC was continued on the same test area previously assigned to receive it. The primary outcome measure was the change in local Scoring Atopic Dermatitis (SCORAD) index between day 1 and 3 based on investigators' assessment. Secondary measures of lesion severity included changes in the local patientoriented SCORAD index, pruritus intensity according to subjects' assessments, and global assessments by subjects and investigators.

Results: The study included 54 subjects. The change in investigator-observed local SCORAD index between day 1 and 3 was $-14.4 \%$ with TCS alone and $-24.5 \%$ for TCS + MDREC $(p=0.0005)$. Between baseline and the end of the treatment phase, all secondary measures of lesion severity decreased more with the combined TCS + MDREC treatment than with the TCS cream alone. The MDREC also reduced the relapse of $\mathrm{AD}$ lesions during the maintenance phase. Tolerability was very good, and the product was well accepted by subjects.

Conclusion: These results support using the MDREC in association with TCS during AD flares and as a maintenance therapy after treatment with TCS has stopped.

Funding: Laboratoires

Dermatologiques 
Keywords: Atopic dermatitis; Clinical trial; Corticosteroid; Dermatology; Dexyane MeD; Emollient; Topical

\section{INTRODUCTION}

Atopic dermatitis (AD) is a disease in which lesions go through periods of remission and flare [1]. During flares, patients have pruritic lesions with erythematous papules, vesicles, excoriations, and serous exudates. Patients with AD also suffer from xerosis (dry skin) and lichenification. AD compromises the skin's barrier function, leaving it susceptible to environmental irritants and allergens [1, 2]. It is also a significant burden for sufferers because it can deprive them of sleep, limit school and work performance, and be expensive and time-consuming to treat [3].

Emollients are recommended as maintenance therapy for $\mathrm{AD}$, and some are used in combination with topical corticosteroids (TCS) during AD flares [4-6]. Medical device repairing emollient creams (MDRECs) act primarily by improving hydration and by providing a physical shield to help restore the skin's barrier function [6]. In Europe, MDRECs are considered class IIa medical devices, meaning that they are non-invasive devices that come into contact with injured skin and which are intended to manage the micro-environment of a wound [7]. As such, unlike cosmetics, they can be applied directly to damaged skin. Maintenance therapy with emollients can reduce $\mathrm{AD}$ flares and the need for TCS [8-11], but evidence of their clinical efficacy when used in combination with TCS is limited.

In the study reported here, we evaluated the added clinical benefit and tolerability of a MDREC when used in association with a moderately potent TCS for adults with moderate to severe AD. The MDREC tested contains a natural polysaccharide mesh that protects and hydrates the skin and is designed to repair and protect the skin barrier alone or in combination with TCS of individuals aged $\geq 3$ months.

\section{METHODS}

\section{Study Design and Ethics}

This was a multicenter, open-label, intra-individual randomized controlled study in adults with moderate to severe AD (EudraCT no. 2014-002194-10). The study was performed at four clinics in Germany between November 2014 and July 2015 . The primary objective was to evaluate the added clinical benefit of the MDREC (Dexyane MeD) when used in association with a moderately potent TCS.

The study protocol was approved by the Independent Ethics Committee of Schleswig-Holstein and authorized by the Bundesinstitut für Arzneimittel und Medizinprodukte of Germany. The study was performed in accordance with the principles stated in the Declaration of Helsinki, ISO 14155:2011, and national regulations. All participating study subjects provided written informed consent.

\section{Subjects}

Adults aged 18-65 years were considered eligible for enrollment if they had a Fitzpatrick skin type of I-IV and a diagnosis of AD for at least 1 year with moderate to severe $\mathrm{AD}$ lesions on both arms or both popliteal fossae. Subjects included in the study had to have a Local Investigator's Global Assessment (L-IGA) severity score of 3 or 4 on a scale of $0-5$ (see section "Assessments") and a local Scoring Atopic Dermatitis (L-SCORAD) index of $\geq 6$ on a scale from 0 to 18 , with moderate or severe xerosis as indicated by a xerosis score of $\geq 2$ on a scale of $0-3$. Lesions on the two arms or popliteal fossae had to be symmetrical, as indicated by a $\leq 1$ point difference in each component of the L-SCORAD index and $\leq 3$ points of difference in the total L-SCORAD index, and the size of the two affected areas had to be similar according to the investigator's judgment. Women who were pregnant or breastfeeding were excluded from the study, and all women enrolled had to be using an effective form of contraception. Subjects were excluded from the study if they had one of more of the following: (1) a non-AD 
lesion, skin disease, or infection on the test areas that could interfere with the study assessments; (2) a disease considered by the investigator to be hazardous for the subject or incompatible with the study; (3) immunosuppression or immunodeficiency; (4) on-going allergen re-introduction or re-introduction diet within the framework of an avoidance regimen; (5) a history of hypersensitivity or intolerance to any component of the TCS or the MDREC. Subjects also could not have been receiving phototherapy within 4 weeks prior to inclusion or during the study, and they could not be taking systemic immunosuppressives within 4 weeks before the study or planned during the study, systemic corticosteroids within 2 weeks before or during the study, systemic antihistamines established or modified within 2 weeks before the study, systemic antibiotics within 1 week before or during the study, topical immunomodulators, non-steroidal anti-inflammatories, corticosteroids, antihistamines, antibiotics, or antiseptics on the test areas within 5 days before or during the study, or skincare products on the test areas within 2 days before inclusion in the study or have washed lesions with water or any cleansing product within $4 \mathrm{~h}$ before inclusion in the study.

\section{Materials}

The MDREC (Dexyane $\mathrm{MeD}^{\circledR}$ ) was produced by Laboratoires Dermatologiques Ducray, Pierre Fabre France and contained glycyrrhetinic $(\beta)$ acid, hydroxydecenoic acid, cetyl alcohol, white beeswax, glyceryl stearate SG, glyceryl stearate/ PEG-100 stearate, Shea butter, capric caprylic triglyceride $30 / 70$, polysorbate 60 , sclerotium gum, polyacrylate-13, glycerin, propylene glycol, 1,3-butanediol, disodium ethylene diamine tetraacetic acid, citric acid monohydrate, guanidine carbonate, capryl glycol, and water. The TCS cream containing $1 \%$ desonide $\left(\right.$ LOCAPRED $^{\circledR}$ ) was from Pierre Fabre France (Castres, France).

\section{Study Conduct}

For each subject, the test areas (arms or popliteal fossae) were randomized 1:1 using a computer-generated list to 10 days of twicedaily application of the MDREC combined with daily application of TCS cream or to 10 days of daily application of TCS cream alone. During this initial "TCS treatment" phase, subjects who had worsening of the test areas necessitating a change of therapy on at least one test area were withdrawn from the study. Subjects were included in a following 2-week "maintenance" phase if, at the end of the TCS treatment phase, the investigator considered that the $\mathrm{AD}$ on at least one test area had sufficiently improved so that the treatment was no longer needed. If treatment was still needed on both test areas at the end of the first period, the subject did not enter the maintenance phase. During the maintenance phase, subjects had to stop treatment with the TCS cream but continued twicedaily application of the MDREC on the same test area previously assigned to receive it.

\section{Assessments}

The primary outcome measure was the change in L-SCORAD index (measured by investigators) between baseline (day 1 ) and 2 days after applying the MDREC (day 3). The L-SCORAD index included the intensity of the six objective signs of the full SCORAD index (erythema, edema/papulation, oozing/crusts, excoriation, lichenification, and dryness) [12]. Secondary outcome measures assessed by investigators included: (1) the L-IGA severity score, scored on a 6-point scale, where $0=$ clear (no inflammatory signs of AD), $1=$ almost clear (just perceptible erythema and just perceptible papulation/infiltration), $2=$ mild disease (mild erythema and mild papulation/infiltration), $3=$ moderate disease (moderate erythema and moderate papulation/infiltration), $4=$ severe disease (severe erythema and severe papulation/infiltration), and $5=$ very severe disease (severe erythema and severe papulation/infiltration with oozing/crusting); (2) transepidermal water loss (TEWL) on the lesions, measured by Aquaflux (Biox Systems, London, UK); (3) local tolerability, scored on a 5-point scale where $1=$ excellent tolerance, $2=$ very good tolerance, $3=$ good tolerance, $4=$ moderate tolerance, and $5=$ bad tolerance. Secondary outcome measures 
assessed by the subjects themselves included (1) pruritus intensity, scored on a 10-point visual analogue scale, ranging from $0=$ none to $10=$ most severe; (2) local patient global assessment compared to baseline (L-PGA), scored using a 5-point scale where $0=$ worse, $1=$ no change, $2=$ slight improvement, 3 = marked improvement, $4=$ total resolution; (3) lesion severity, scored using the local patient-oriented SCORAD (L-PO-SCORAD) index, which includes the intensity of the six objective signs of the full PO-SCORAD index (erythema, edema/papulation, oozing/crusts, excoriation, lichenification, and dryness) [13]. At the end of the study, subjects rated their global satisfaction with the MDREC as "disliked it a lot," "disliked it," "liked it moderately," "liked it," or "liked it a lot" and using a 10-point scale ranging from 0 for "like a lot" to 10 for "dislike a lot."

\section{Study Size Estimation}

A sample size of 49 available subjects was required to achieve $90 \%$ power based on a two-sided type-I error of 0.05 and assuming a $60 \%$ reduction of the primary efficacy assessment (change in L-SCORAD index between baseline and day 3) for the arm receiving TCS + MDREC, a 40\% reduction between baseline and day 3 for the arm receiving TCS alone, a mean L-SCORAD at baseline of 9 , and a common standard deviation of 3 . To account for an estimated $10 \%$ of non-assessable subjects, 54 subjects were to be included.

\section{Statistical Analysis}

The primary efficacy assessment was an analysis of covariance of the primary outcome measure (change in L-SCORAD index between baseline and day 3 ) in the full analysis set (all subjects included) with test area, product, and sequence as fixed factors, subject as random factor, and value at baseline as a covariate. In cases where at least one evaluation was missing after the first post-product application assessment, the last observation carried forward method was used to replace missing data. Sensitivity analysis for the primary efficacy assessment was performed in the per-protocol set (all subjects without any major protocol deviation or other source of bias for primary analyses and with sufficient study product exposure).

Secondary outcome measures were analyzed in the full analysis set. These included (1) the change in L-SCORAD index from baseline analyzed using a mixed model for repeated measures with test area, visit, product, and product $\times$ test area interaction as fixed factors, subject as random factor, and the L-SCORAD index at baseline as a covariate; (2) area under the curve between baseline and day 10 for each component of the L-SCORAD index (erythema, edema/papulation, oozing/crusts, excoriation, lichenification, and dryness), L-PO-SCORAD index, L-IGA, L-PGA, pruritus intensity, and TEWL analyzed by analysis of variance with the test area, product, and product $\times$ test area interaction as fixed factors and subject as a random factor; (3) change between baseline and day 3 in pruritus severity and L-PGA by Wilcoxon signed-rank test; (4) change between baseline and day 24 in L-SCORAD index, L-IGA, pruritus intensity, L-PO-SCORAD index, and L-PGA by Wilcoxon signed-rank test; (5) time to relapse analyzed using the Kaplan-Meier method and the log-rank model; (6) percentage without relapse compared by McNemar's test. For the latter two analyses, relapse was defined as the need to restart the subject's usual treatment or other product due to worsening of AD. For secondary outcome measures, missing data were not replaced.

Statistical analysis was performed using SAS ${ }^{\circledR}$ version 9.3 (SAS Institute, Cary, NC, USA). All statistical tests were two-tailed with a type-I error of 0.05 .

\section{RESULTS}

Fifty-four subjects were enrolled in the study between 24 November 2014 and 17 June 2015, and the study was completed on 9 July 2015 (Fig. 1). All subjects remained in the study to at least the end of the TCS treatment phase (day 10) and therefore provided data for the primary efficacy assessment.

\section{Subject Baseline Characteristics}

The 54 subjects enrolled in this study ranged from 18 to 63 years of age (mean 


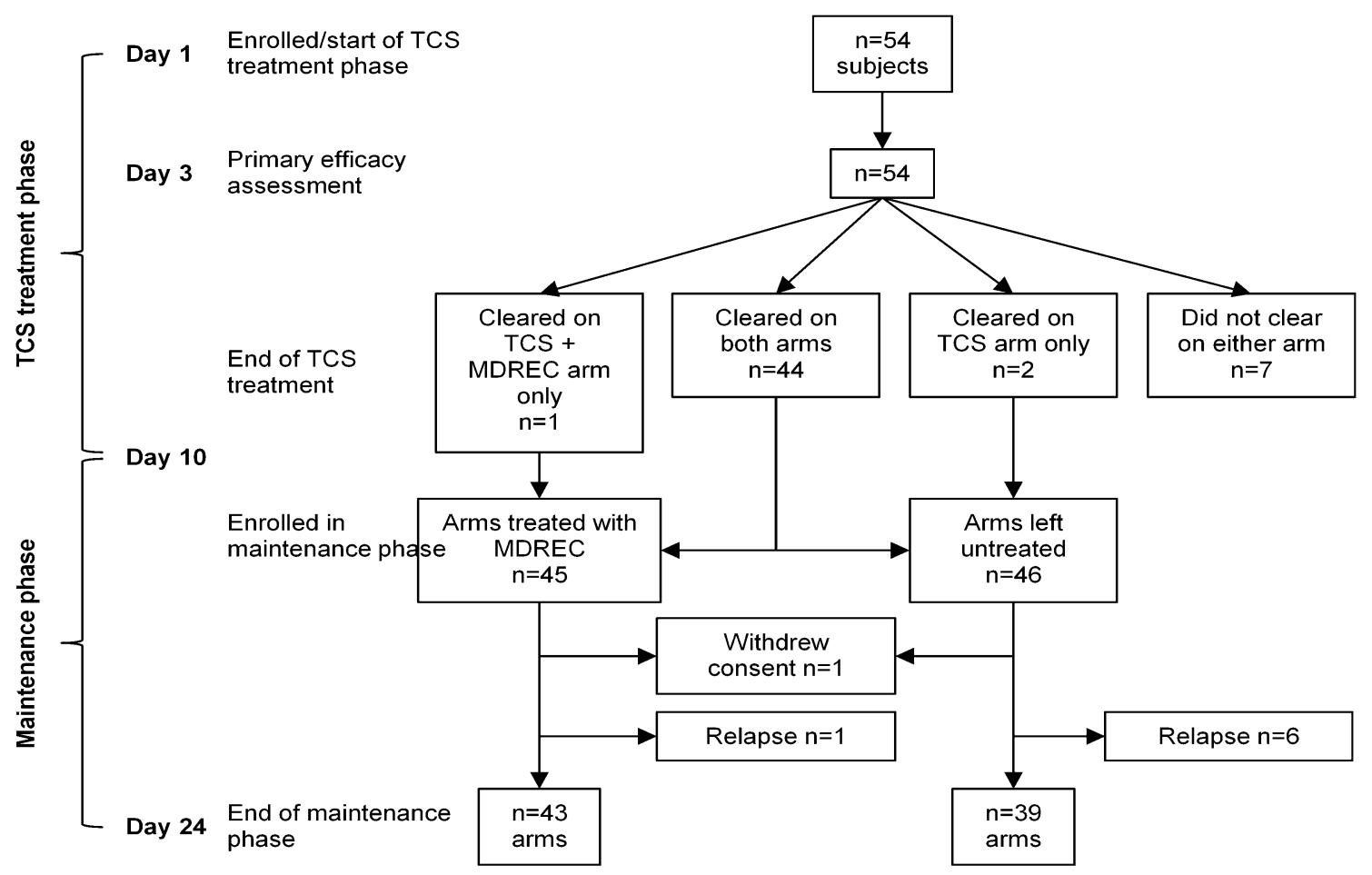

Fig. 1 Flow chart of study design and disposition of subjects. For each subject, the test areas were randomized 1:1 to 10 days of twice-daily application of the topical corticosteroid $(T C S)+$ medical device repairing emollient cream (MDREC) or the TCS alone. Subjects were included in a following 2 -week maintenance phase if, at the end of the TCS treatment phase, the investigator

$31.7 \pm 12.1$ years). Just over half of the subjects were women $(n=31 ; 57.4 \%)$. The most common skin type was Fitzpatrick type III $(n=26$; $48.1 \%)$, followed by type II $(n=16 ; 29.6 \%)$, type IV $(n=11 ; 20.4 \%)$, and type I $(n=1$; $1.9 \%)$. Most subjects $(n=38 ; 70.4 \%)$ had a family history of AD.

On average, subjects had been diagnosed with $\mathrm{AD}$ for $27.1 \pm 12.2$ (range 1-63) years before inclusion in this study. Most subjects $(n=45$; $83.3 \%$ ) had at least one concomitant disease, known allergy, or both when included in the study. None of these conditions were considered to interfere with the study assessments.

Most of the subjects $(n=49 ; 90.7 \%)$ had applied $\mathrm{AD}$ treatments during the past year, in most cases $(n=45 ; 83.3 \%)$ topical treatments only. None of the subjects had taken only systemic treatments, although a few $(n=3 ; 5.6 \%)$ had been treated with both topical treatments considered that the atopic dermatitis on at least one of the two test areas had sufficiently improved so that the treatment was no longer needed. During the maintenance phase, subjects stopped treatment with the TCS cream but continued twice-daily application of the MDREC on the same test area previously assigned to receive it

and systemic treatments and one (1.9\%) had been treated with phototherapy in addition to oral and topical treatments.

Approximately two-thirds of the AD lesions included in the study were of moderate intensity, and the remainder were of severe intensity (Table 1). Lesion severity appeared to be balanced between test areas randomized to TCS alone and those randomized to treatment with TCS + MDREC.

\section{Changes in Clinical and Functional Signs During the TCS Treatment Phase (Days 1-10)}

Changes in L-SCORAD Scores During the TCS Treatment Phase

The primary outcome measure, i.e., change in L-SCORAD between baseline (day 1) and day 3, 
was $-14.4 \%$ for the TCS alone treatment and $-24.5 \%$ in the combined treatment of TCS + MDREC $(p=0.0005)$ (Fig. 2a). This difference was confirmed by a sensitivity analysis limited to subjects completing the study according to protocol $(p=0.0027$; data not shown). A difference between the TCS and TCS + MDREC treatments was also found over the full treatment phase ( $p=0.0002$ ) (Fig. 3a).

\section{Changes in L-SCORAD Sub-Scores During the TCS Treatment Phase}

Between baseline and the end of the TCS treatment phase, the xerosis $(p<0.0001)$ and edema/papulation $(p=0.0161)$ sub-scores of the L-SCORAD index decreased more with the combined TCS + MDREC treatment than with TCS alone. A difference between treatments was not detected for erythema ( $p=0.0894)$, oozing/ crusts $(p=0.1835)$, excoriation $(p=0.1201)$, or lichenification $(p=0.0844)$ (data not shown).

\section{Changes in Other Investigator Assessments During the Treatment Phase}

As assessed by the L-IGA score, the change in lesion severity from baseline was greater with the combined TCS + MDREC treatment than with TCS alone when assessed at day 3 ( $p=0.0187$ ) (Fig. 2b). A difference between the TCS and TCS + MDREC treatments was also found over the full TCS treatment phase $(p=0.0197)$ (Fig. 3b).

TEWL decreased between baseline and the end of the treatment phase to a similar extent with both the TCS + MDREC and TCS alone treatments ( $p=0.4940$; data not shown).

\section{Changes in Subject Assessments During the TCS Treatment Phase}

According to subjects' assessment, the change in pruritus intensity from baseline was greater with the combined TCS + MDREC treatment than with TCS alone when assessed at day 3 $(p=0.0188)$ (Fig. 2c), although a difference was not found between the TCS and TCS + MDREC treatments over the full treatment phase $(p=0.0623$ ) (Fig. 3c). Also, during the full TCS

Table 1 Severity of test lesions at baseline

\begin{tabular}{llll}
\hline Evaluator & Assessment & TCS only $(\boldsymbol{n}=\mathbf{5 4})$ & TCS + MDREC $(\boldsymbol{n}=\mathbf{5 4})$ \\
\hline Investigator & L-IGA category & & \\
& Moderate $($ score $=3)$ & $35(64.8 \%)$ & $33(61.1 \%)$ \\
& Severe $($ score $=4)$ & $19(35.2 \%)$ & $21(38.9 \%)$ \\
& L-IGA score & $3.39 \pm 0.49$ \\
& L-SCORAD index & & $10.20 \pm 2.45$ \\
& TEWL $\left(\mathrm{g} / \mathrm{m}^{2} / \mathrm{h}\right)^{\mathrm{c}}$ & $10.43 \pm 2.40$ & $46.13 \pm 19.79$ \\
Subjects & L-PO-SCORAD index & $43.77 \pm 20.15$ & $7.35 \pm 2.71$ \\
& Pruritus intensity & \\
& & $7.59 \pm 2.38$ & $4.14 \pm 2.42$ \\
\hline
\end{tabular}

Values in table are presented as the number of subjects with the percentage in parenthesis or as the mean \pm standard deviation (SD)

L-IGA Local Investigator's Global Assessment, L-PO-SCORAD local patient-observed Scoring Atopic Dermatitis, $L$ SCORAD local SCORAD, MDREC medical device repairing emollient cream, TCS topical corticosteroid, TEWL transepidermal water loss

${ }^{a}$ Scored on a 6-point scale from 0 for clear (no inflammatory signs of atopic dermatitis) to 5 for very severe disease

b Scores range from 0 for no signs to 18 for most severe

${ }^{c}$ Measured by Aquaflux (Biox Systems, London, UK)

${ }^{\mathrm{d}}$ Scored on a 10-point visual analogue scale, ranging from $0=$ none to $10=$ most severe 

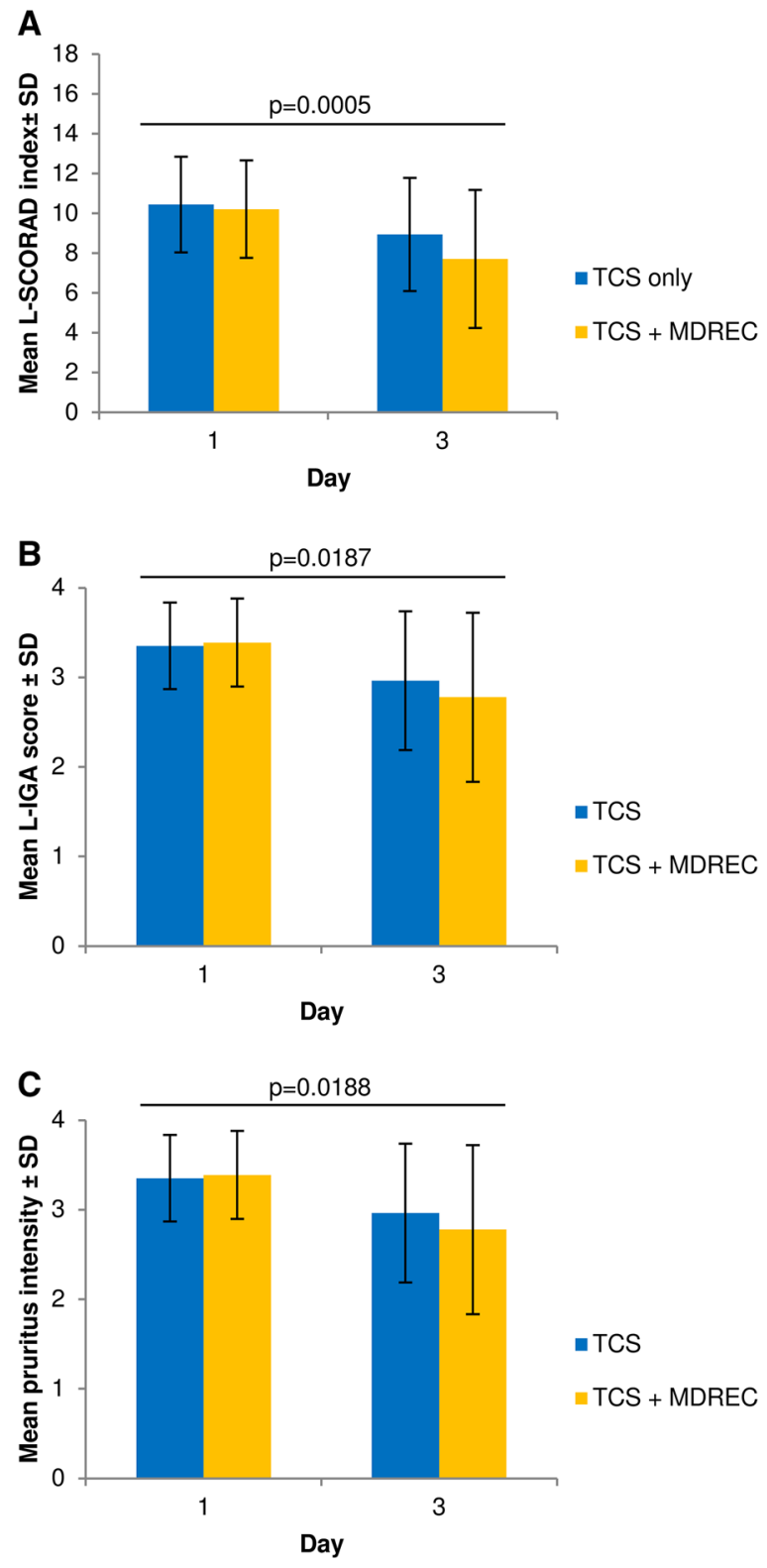

Fig. 2 Change in the local Scoring Atopic Dermatitis ( $L$ $S C O R A D)$ index (a), local Investigator's Global Assessment $(L-I G A)(\mathbf{b})$, and subject-assessed pruritus (c) between day 1 (baseline) and day 3 during the TCS treatment phase. $P$ values were for comparisons of mean changes from baseline and were determined by analysis of covariance for L-SCORAD index (a) and by Wilcoxon signed-rank test for L-IGA (b) and subject-assessed pruritus (c). SD Standard deviation

treatment phase, lesions improved more with the TCS + MDREC treatment than with TCS alone according to the L-PGA score $(p=0.0001)$ and the L-PO-SCORAD ( $p=0.0008)$ (Fig. 3d, e).

\section{Changes in Clinical and Functional Signs During the Maintenance Phase (Days 11-24)}

Forty-seven subjects whose lesions cleared on at least one test area were included in the 2-week maintenance phase (days 10-24) (Fig. 1). This included one subject whose lesions cleared only on the test area receiving TCS + MDREC, two whose lesions cleared only on the test area receiving TCS alone, and 44 subjects whose lesions cleared on both arms. Therefore, 45 test areas received MDREC and 46 test areas were left untreated during the maintenance phase. One subject who had both test areas included in the maintenance phase voluntarily withdrew from the study before day 24 for a personal reason unrelated to an adverse event (AE). Also, during the maintenance phase, relapse occurred on one test area that received MDREC alone and on six test areas that were left untreated. Thus, 43 test areas receiving the MDREC and 39 test areas that did not receive it continued receiving/no receiving the MDREC to the end of the maintenance phase on day 24 .

\section{Investigator Assessments during the Maintenance Phase}

According to investigator assessments (LSCORAD and L-IGA), between baseline and the end of the maintenance phase (day 24), lesions improved more in the TCS + MDREC study arm than in the TCS alone study arm $(-61.71 \%$ vs. $-45.16 \% \quad[p=0.0056]$ for L-SCORAD and $-56.08 \%$ vs. $-43.39 \%$ [ $p=0.0195]$ for L-IGA) (Table 2).

\section{Subject Assessments During the Maintenance Phase}

According to study subjects, between baseline and the end of the maintenance phase, pruritus intensity decreased more for the TCS + MDREC study arm $(-69.15 \%)$ than for the TCS alone study arm $(-51.94 \%) \quad(p=0.0014)$ (Table 2). Subjects also reported that lesion severity at the end of the maintenance phase, as measured by L-PGA, was significantly better for the TCS + MDREC study arm (26.2\%) than for the TCS alone study arm $(7.9 \%) \quad(p=0.0002)$. 

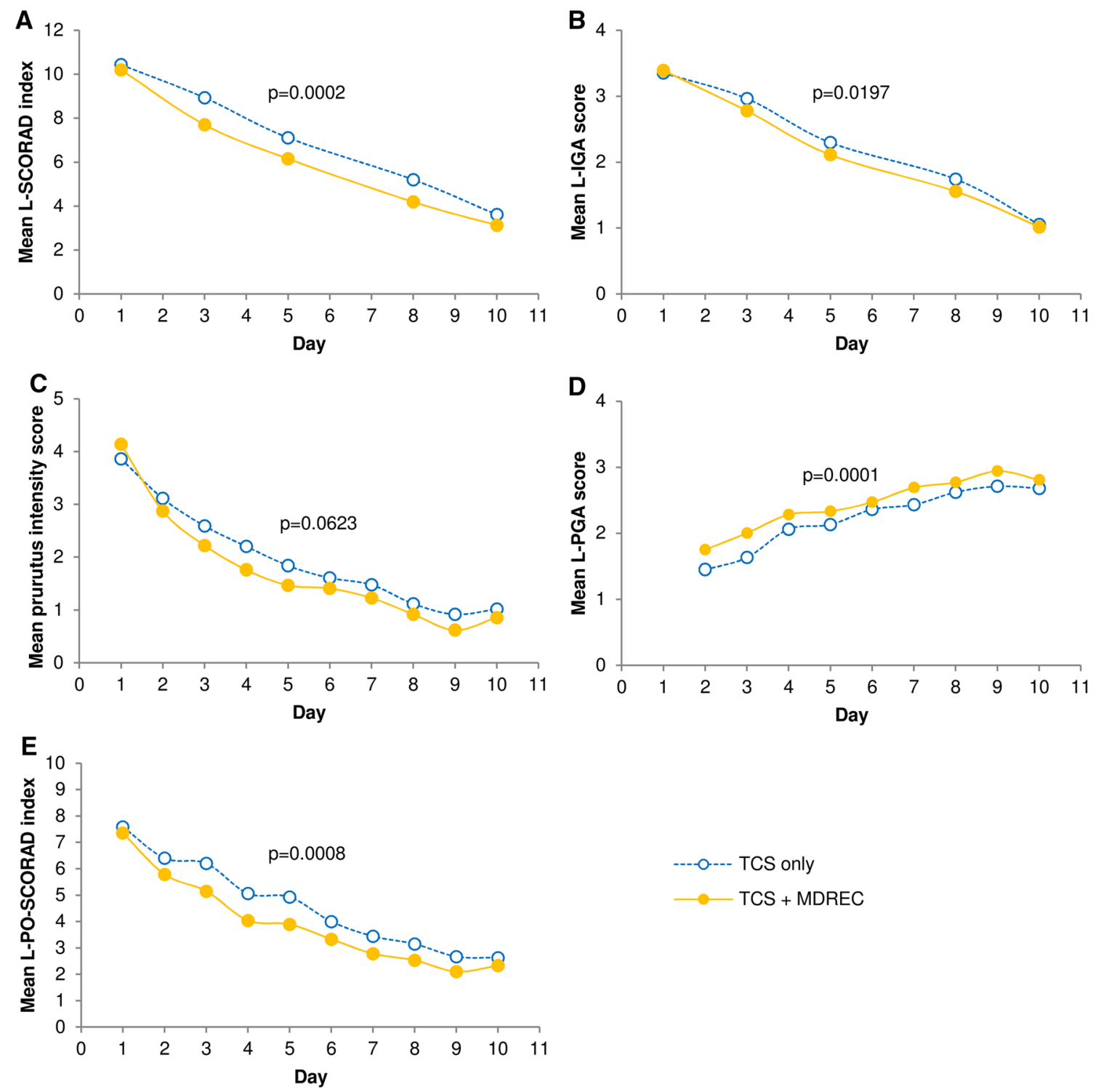

Fig. 3 Change in L-SCORAD index (a), L-IGA (b), subject-assessed pruritus (c), local patient global assessment compared to baseline $(L-P G A)(\mathbf{d})$, and local patientobserved SCORAD (L-PO-SCORAD) index (e) between day 1 (baseline) and the end of the TCS treatment phase (day 10). $P$ values for differences in L-SCORAD index

However, the change in L-PO-SCORAD between baseline and the end of the maintenance phase was not significantly different between the TCS + MDREC study arm $(-56.66 \%)$ and the TCS alone study arm $(-41.58 \%)(p=0.0577)$.

over the TCS treatment phase (a) were calculated by mixed model for repeated measures; for all other assessments $(\mathbf{b}-\mathbf{f}), p$ values were calculated for differences in area under the curve by analysis of variance

Incidence of Relapse During the Maintenance Phase

Relapse during the maintenance phase, as indicated by a need for therapy for worsening of $\mathrm{AD}$, occurred in $2.3 \%$ ( 1 of 44 ) study arms receiving MDREC and 13.3\% (6 of 45) not receiving it $(p<0.001)$. The mean number of 
Table 2 Change in clinical and functional signs of atopic dermatitis at the end of the maintenance phase (day 24)

\begin{tabular}{|c|c|c|c|c|}
\hline Evaluator & Assessment $^{\mathrm{a}}$ & $\begin{array}{l}\text { TCS alone study arm } \\
(n=39)\end{array}$ & $\begin{array}{l}\text { TCS + MDREC study arm } \\
(n=43)\end{array}$ & $P$ value ${ }^{b}$ \\
\hline \multirow[t]{2}{*}{ Investigator } & L-SCORAD index & $-4.74 \pm 3.42$ & $-6.05 \pm 2.95$ & 0.0056 \\
\hline & L-IGA score & $-1.41 \pm 1.21$ & $-1.84 \pm 1.19$ & 0.0195 \\
\hline \multirow[t]{8}{*}{ Subjects } & Pruritus intensity & $-1.81 \pm 2.41$ & $-2.81 \pm 2.68$ & 0.0014 \\
\hline & L-PO-SCORAD index & $-2.85 \pm 3.40$ & $-3.81 \pm 3.57$ & 0.0577 \\
\hline & L-PGA score & & & \\
\hline & Worse $($ score $=0)$ & $2(5.3 \%)$ & $2(4.8 \%)$ & 0.0002 \\
\hline & No change $($ score $=1)$ & $7(18.4 \%)$ & $1(2.4 \%)$ & \\
\hline & $\begin{array}{l}\text { Slight improvement } \\
(\text { score }=2)\end{array}$ & $18(47.4 \%)$ & $19(45.2 \%)$ & \\
\hline & $\begin{array}{l}\text { Marked improvement } \\
(\text { score }=3)\end{array}$ & $8(21.1 \%)$ & $9(21.4 \%)$ & \\
\hline & Total resolution $($ score $=4)$ & $3(7.9 \%)$ & $11(26.2 \%)$ & \\
\hline
\end{tabular}

Values in table are presented as the number of subjects with the percentage in parenthesis or as the mean $\pm S D$

${ }^{a} L-P G A$ (local patient's global assessment) was scored on a 5-point scale from 0 for worse lesions to 4 for total resolution of lesions. For explanation of all other indexes/scores, see footnotes to Table 1

b $P$ values were determined by Wilcoxon sign-rank test

days in the maintenance phase (i.e., before relapse) was not significantly different between study arms receiving MDREC $(12.47 \pm 3.03$ days) and those not receiving it $(13.00 \pm 2.32$ days; $p=0.25)$. Time to relapse was also not significantly different between study arms (21.38 \pm 0.41 days vs. not calculable; $p=0.0562$ ).

\section{Safety and Tolerability}

\section{Adverse events}

Ten subjects had AEs suspected to be related to the MDREC only. These included six subjects who reported a mild burning sensation on the test lesion lasting 1-2 days after application, two subjects who reported mild application-site pruritus lasting 1 day, and two subjects who reported mild exacerbation of $\mathrm{AD}$ during the maintenance phase that did not resolve before the end of the study. None of these AEs led to discontinuation of MDREC or corrective treatments. Also, no serious AEs or other AEs resulting in discontinuation were reported.

\section{Investigator-Assessed Tolerability}

When assessed at the end of the TCS treatment phase on day 10, investigators rated global local tolerability of the MDREC as very good $(n=3$; $5.6 \%)$ to excellent $(n=51 ; 94.4 \%)$ for all subjects. When assessed again at the end of the maintenance phase on day 24, global local tolerability was rated very good $(n=1 ; 2.3 \%)$ to excellent $(n=42 ; 97.7 \%)$.

\section{Subject Satisfaction}

According to questionnaires, $81.5 \%(44 / 54)$ of subjects reported that they liked the MDREC, and the remainder reported that they moderately liked it. The mean satisfaction score, assessed on a 10-point scale, was $7.45 \pm 1.65$, and $94.3 \%(50 / 53)$ of subjects assigned a score of $>5$. 


\section{DISCUSSION}

Medical device repairing emollient creams improve hydration and provide a physical shield for damaged skin [6]. As non-invasive devices that come into contact with injured skin with the aim to manage the micro-environment of a wound, they can be considered class IIa medical devices [14]. Unlike cosmetics, they can be applied to damaged skin, such as $\mathrm{AD}$ lesions during flares, but little evidence is available to support their clinical efficacy when used in association with TCS.

In this study, we showed that a MDREC accelerated improvement in lesions in adults with moderate to severe $\mathrm{AD}$ when used in association with $0.1 \%$ desonide, a moderately potent TCS. The study also showed that the MDREC slowed the return of AD lesions after the TCS had been stopped.

Both investigators and study subjects reported more rapid improvement on arms receiving the MDREC, as shown especially by a significant difference in the primary outcome measure, i.e., the change in the L-SCORAD between baseline and day 3. According to several additional measures by investigators and subjects, this more rapid improvement with TCS + MDREC was maintained over the 10-day TCS treatment phase. These effects appeared to be largely due to an added benefit of the MDREC in reducing xerosis and edema/papulation. This result agrees with the findings of a randomized, investigator-masked study in $\mathrm{AD}$ patients aged $\geq 6$ years, which showed that adding a moisturizing regimen to TCS treatment improved symptom scores, PGA, and most individual efficacy variables [15]. Two other studies in children showed that when co-applied, an emollient can reduce the need for TCS $[16,17]$.

The MDREC also appeared to slow relapse of AD lesions after the TCS was stopped, although we did not detect a significant effect on the time to relapse, probably because the 2-week maintenance phase was too short. Increasing the follow-up time in the current study to detect a significant difference in time to relapse would have been difficult because of the intra-individual randomized design. An effect on the time to relapse would be more easily detected in a longer randomized controlled study including two separate groups.

Mild burning or itching suspected to be related to the treatment was reported by some subjects. These symptoms are common when TCS and other products are applied directly to diseased skin $[1,2,18]$. The only other events considered to be possibly related to the treatment was mild exacerbation of AD in two subjects, but this did not lead to a change in medication or discontinuation of the study. These events therefore do not appear to be a significant concern and, moreover, subjects appreciated the MDREC and investigators considered it well tolerated.

This study used a robust intra-individual randomized design with a validated, investigator-assessed score as a primary efficacy endpoint and several supporting secondary endpoints. We did not include MDREC and placebo controls in the study because this would have required withholding treatment from $\mathrm{AD}$ sufferers. This absence of controls precluded us from determining to what extent the mild burning and itching were due to the MDREC. Also, although this study demonstrated a significant effect of the MDREC during and following $\mathrm{AD}$ flares, a longer study is needed to determine long-term effects and whether the MDREC increased the time to relapse. Finally, we did not differentiate between intrinsic and extrinsic cases of AD. Given that the MDREC works primarily by providing a physical barrier, we suspect that it would help regardless of the type of atopy.

\section{CONCLUSIONS}

The primary and several secondary assessments provided strong evidence that the MDREC provides an added clinical benefit when used in association with TCS for treating AD flares and for maintenance after stopping TCS treatment. The results of this study also strengthen the evidence base supporting the use of MDRECs in association with TCS for patients with AD. 


\section{ACKNOWLEDGEMENTS}

Funding. Sponsorship for this study and article processing charges were funded by Laboratoires Dermatologiques Ducray, Pierre Fabre, France. All authors had full access to all of the data in this study and take complete responsibility for the integrity of the data and accuracy of the data analysis.

Authorship. All named authors meet the International Committee of Medical Journal Editors (ICMJE) criteria for authorship of this manuscript, take responsibility for the integrity of the work as a whole, and have given final approval to the version to be published.

\section{Medical Writing and Other Assis-} tance. Medical writing in the preparation of this manuscript was provided by Dr. Phillip Leventhal of 4Clinics (Paris, France). Financial support for this assistance was provided by Pierre-Fabre Dermo-Cosmetique. The authors would like to thank the following people: Professor Carle Paul (Head of the Department of Dermatology, University Hospital of Toulouse) for thoughtful discussions about the study design; Valerie Mengeaud (Medical Director, Laboratoires Dermatologiques Ducray, Pierre Fabre, France) for critically reviewing the manuscript; Angela Hougardy (proDERM) for regulatory support; Sylva Bolduan (GCP Service) for monitoring the study; Christophe Lauze, Pierre Fabre Biometry for statistical support; the Biostatem team for the data management and statistical analyses; Sabine Biais (Pierre Fabre) for formulating the MDREC; the study investigators (Professor Klaus-Peter Wilhelm, Professor Regina Fölster-Holst, Professor Diamant Thaci, and Dr Swarna Ekanayake-Bohlig) and their coinvestigators for recruiting and evaluating the patients.

Disclosures. During this study, Ana Beatris Rossi was an employee of Pierre-Fabre DermoCosmetique. Adeline Bacquey is an employee of Pierre-Fabre Dermo-Cosmetique. Thérèse Nocera is an employee of Pierre-Fabre Dermo-
Cosmetique. Marie-Dominique Thouvenin is an employee of Pierre-Fabre Dermo-Cosmetique.

Compliance with Ethics Guidelines. All procedures followed were in accordance with the ethical standards of the responsible committee on human experimentation (institutional and national) and with the Helskinki Declaration of 1964, as revised in 2013. Informed consent was obtained from all patients for being included in this study.

Data Availability. The datasets analyzed during the current study will not be shared.

Open Access. This article is distributed under the terms of the Creative Commons Attribution-NonCommercial 4.0 International License (http://creativecommons.org/licenses/ by-nc/4.0/), which permits any noncommercial use, distribution, and reproduction in any medium, provided you give appropriate credit to the original author(s) and the source, provide a link to the Creative Commons license, and indicate if changes were made.

\section{REFERENCES}

1. Weidinger S, Novak N. Atopic dermatitis. Lancet. 2016;387(10023):1109-22.

2. Lee HJ, Lee SH. Epidermal permeability barrier defects and barrier repair therapy in atopic dermatitis. Allergy Asthma Immunol Res. 2014;6(4):276-87.

3. Blome C, Radtke MA, Eissing L, Augustin M. Quality of life in patients with atopic dermatitis: disease burden, measurement, and treatment benefit. Am J Clin Dermatol. 2016;17(2):163-9.

4. Mohan GC, Lio PA. Comparison of dermatology and allergy guidelines for atopic dermatitis management. JAMA Dermatol. 2015;151(9):1009-13.

5. Vestergaard C, Deleuran M. Advances in the diagnosis and therapeutic management of atopic dermatitis. Drugs. 2014;74(7):757-69.

6. $\mathrm{Ng} \mathrm{JP}$, Liew HM, Ang SB. Use of emollients in atopic dermatitis. J Eur Acad Dermatol Venereol. 2015;29(5):854-7. 
7. European Commission. Manual on bordeline and classification in the community regulatory framework for medical devices. Version 1.18 (12-2017). Brussels: European Commission, 2017.

8. Tiplica GS, Kaszuba A, Malinauskiene L, Konno P, Boralevi F, Garrigue E, et al. Prevention of flares in children with atopic dermatitis with regular use of an emollient containing glycerol and paraffin: a randomized controlled study. Pediatr Dermatol. 2017;34(3):282-9.

9. Mengeaud V, Phulpin C, Bacquey A, Boralevi F, Schmitt AM, Taieb A. An innovative oat-based sterile emollient cream in the maintenance therapy of childhood atopic dermatitis. Pediatr Dermatol. 2015;32(2):208-15.

10. Boralevi F, Saint Aroman M, Delarue A, Raudsepp $\mathrm{H}$, Kaszuba A, Bylaite M, et al. Long-term emollient therapy improves xerosis in children with atopic dermatitis. J Eur Acad Dermatol Venereol. 2014;28(11):1456-62.

11. Grimalt R, Mengeaud V, Cambazard F. The steroidsparing effect of an emollient therapy in infants with atopic dermatitis: a randomized controlled study. Dermatology. 2007;214(1):61-7.

12. European Task Force on Atopic Dermatitis. Severity scoring of atopic dermatitis: the SCORAD index. Consensus report of the European task force on atopic dermatitis. Dermatology. 1993;186(1):23-31.

13. Vourc'h-Jourdain M, Barbarot S, Taieb A, Diepgen $\mathrm{T}$, Ambonati M, Durosier V, et al. Patient-oriented
SCORAD: a self-assessment score in atopic dermatitis. A preliminary feasibility study. Dermatology. 2009;218(3):246-51.

14. European Medicines Agency. Manual on borderline and classification in the community regulatory framework for medical devices, version 1.17 (092015). 2015. http://ec.europa.eu/consumers/ sectors/medical-devices/files/wg_minutes_member_ lists/version1_9_borderline_manual_en.pdf. Accessed 10 May 2017.

15. Hanifin J, Hebert AA, Mays SR, Paller AS, Sherertz $\mathrm{EF}$, Wagner AM, et al. Effects of a low-potency corticosteroid lotion plus a moisturizing regimen in the treatment of atopic dermatitis. Curr Ther Res. 1998;59(4):227-33.

16. Msika P, De Belilovsky C, Piccardi N, Chebassier N, Baudouin C, Chadoutaud B. New emollient with topical corticosteroid-sparing effect in treatment of childhood atopic dermatitis: SCORAD and quality of life improvement. Pediatr Dermatol. 2008;25(6):606-12.

17. Lucky AW, Leach AD, Laskarzewski P, Wenck H. Use of an emollient as a steroid-sparing agent in the treatment of mild to moderate atopic dermatitis in children. Pediatr Dermatol. 1997;14(4):321-4.

18. Hajar T, Leshem YA, Hanifin JM, Nedorost ST, Lio PA, Paller AS, et al. A systematic review of topical corticosteroid withdrawal ("steroid addiction") in patients with atopic dermatitis and other dermatoses. J Am Acad Dermatol. 2015;72(3):541-9. 